\title{
Identification of the $e^{+} e^{-} \rightarrow n \bar{n}$ events in CMD-3 detector
}

\author{
A.N.Amirkhanov ${ }^{1,2, *}$, G.V.Fedotovich ${ }^{1,2}$, S.S.Gribanov ${ }^{1,2}$, I.B.Logashenko ${ }^{1,2}$, A.S.Popov ${ }^{1,2}$, \\ N.M.Ryskulov ${ }^{1}$, and A.E.Ryzhenenkov ${ }^{1,2}$ \\ ${ }^{1}$ Budker Institute of Nuclear Physics SB RAS, Novosibirsk 630090 \\ ${ }^{2}$ Novosibirsk State University, Novosibirsk 630090
}

\begin{abstract}
A large amount of data collected with the CMD-3 detector above the threshold of the $n \bar{n}$ pair allows to measure the cross section of the process $e^{+} e^{-} \rightarrow n \bar{n}$ and the neutron form factor with good accuracy. This task is complicated by a large number of background events and necessity of finding ways to suppress them. A technique of identification for $e^{+} e^{-} \rightarrow n \bar{n}$ events is described.
\end{abstract}

\section{Introduction}

The work presented in this paper is aimed to develop algorithms for identification of the $e^{+} e^{-} \rightarrow n \bar{n}$ process. Identification is complicated because of a large number of background events, mostly cosmic and beam events. A technique of identification of $n \bar{n}$ events described below is using main detector systems and neural network to reduce the background of cosmic events. The main purpose of this task is to measure the cross section of the process after its identification.

Currently the cross section was measured in two experiments - FENICE[1] and SND[2] and the accuracy of the measurement was limited, $\sim 40 \%$ at the FENICE detector and $\sim 20 \%$ at SND.

\section{Collider and detector}

\section{VEPP-2000 and CMD-3 detector}

Since 2010 the CMD-3 detector has been collecting data at the VEPP-2000 $e^{+} e^{-}$collider. CMD-3 is a general-purpose detector designed to study $e^{+} e^{-}$annihilation into hadrons in the center-of-mass energy range from 0.3 up to $2 \mathrm{GeV}$ and described elsewhere[3]. CMD-3 is shown in Fig. 1.

A detector tracking system consists of a cylindrical drift chamber (DC) that has 1218 hexagonal cells with sensitive wires and a double-layer cylindrical multiwire proportional chamber (Z-chamber), both installed inside a thin $\left(0.085 X_{0}\right)$ superconducting solenoid with $1.3 \mathrm{~T}$ magnetic field. A barrel part of an electromagnetic calorimeter that is placed outside of the solenoid comprises a liquid Xenon calorimeter $\left(5.1 X_{0}\right)$ and a calorimeter based on CsI crystals $\left(8.1 X_{0}\right)$. The endcap calorimeter is based on BGO crystals $\left(13.5 X_{0}\right)$. Between the LXe and CsI calorimeters the time-of-flight (TOF) system[4] is placed and outside the detector a muon system is installed.

The data above the $n \bar{n}$ threshold was collected in $2011\left(2.5 \mathrm{pb}^{-1}\right), 2012\left(3.8 \mathrm{pb}^{-1}\right)$ and $2017\left(27 \mathrm{pb}^{-1}\right)$.

\footnotetext{
*email: amirkhanovartem@gmail.com
} 


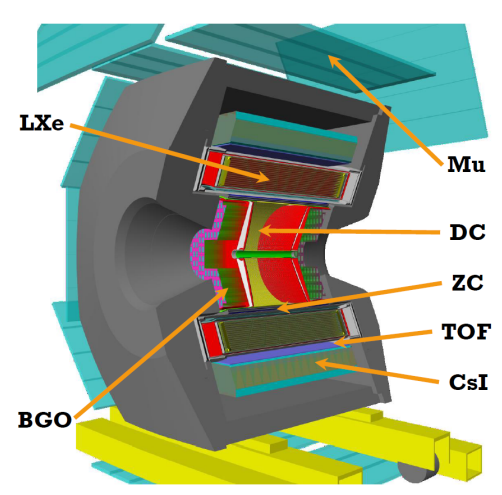

Figure 1: CMD-3 detector.

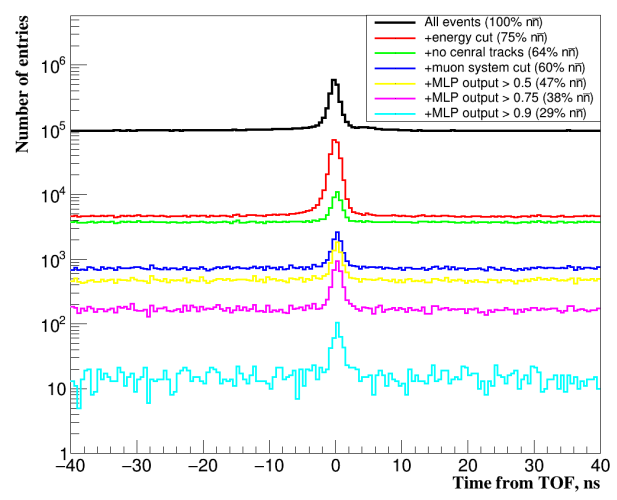

Figure 2: The number of remaining events after different selections.

\section{Time-of-flight system}

A significant part of background events in this analysis can be filtered using various detector systems. Near the production threshold, $n \bar{n}$ events have relatively low velocities and correspondingly a long time of flight through the detector. The average times of annihilation in the calorimeters have a typical delay time with the respect to the beam collision. The final step of selection is the use of the time of flight of $n \bar{n}$ events and the TOF system is used to this end.

TOF was fully upgraded in 2017 and consists of 175 scintillation counters. The light is collected by WLS the fiber, equipped with two SiPMs, one at each end. The time resolution of the system in case of the average time from both SiPM on the plate varies from 0.55 to $0.75 \mathrm{~ns}$ and depends on the counter. When data for all counters in an event is combined, the time resolution is about $330 \mathrm{ps}$.

\section{Data analysis}

\section{$e^{+} e^{-} \rightarrow n \bar{n}$ events}

A neutron passing through the detector systems is usually not detected. An antineutron annihilates in the calorimeter and as a result of the interaction a number of new particles is born, which can be detected. The energy deposition in the calorimeter for antineutron events has a "star"-like symmetric shape.

Near the production threshold antineutrons have relatively low velocities and correspondingly a long time of flight through the detector. The average times of annihilation in the calorimeters have a time delay with respect to the beam collision, which can be used for the identification of $e^{+} e^{-} \rightarrow n \bar{n}$ events. For example, the time delay is $\sim 4-12$ ns with respect to the beam crossing at $E_{\text {beam }}=980 \mathrm{MeV}$.

The main source of background events is cosmic events which come at random times and products of the $e^{+} e^{-}$annihilation which come promptly after a collision. The shape of the energy deposition in the calorimeter for most of the cosmic events has a stretched form as opposed to an antineutron. The cosmic background is $\sim 10^{4}$ times larger than the expected $n \bar{n}$ signal.

\section{Event Selection}

One of the main selection criteria is the shape of the cluster in the calorimeters. Momenta of inertia were calculated for the biggest (in terms of energy) cluster in the event. Close values 


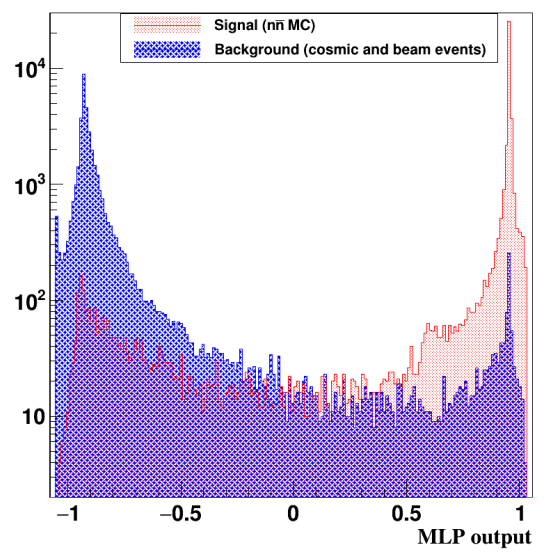

Figure 3: MLP output for $n \bar{n}$ events.

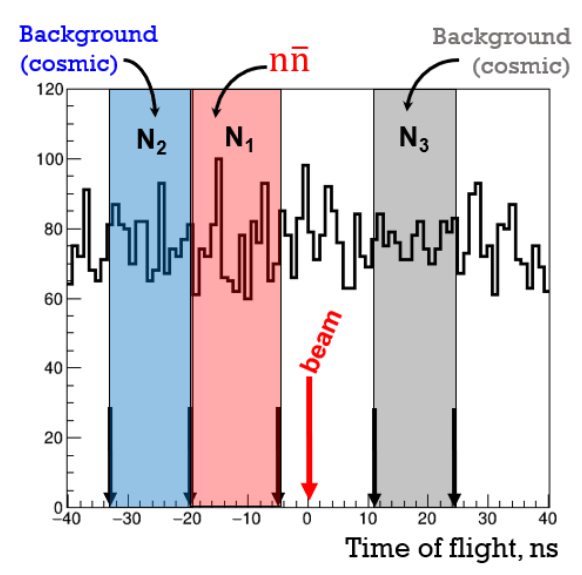

Figure 4: Counting signal events. $\mathrm{N}($ signal $)=N_{1}-N_{3}$ and $\mathrm{N}($ control $)=N_{2}-N_{3}$. Borders depend from the $E_{\text {beam }}$.

of momenta correspond to an antineutron event ("star"-like, symmetric shape of the cluster), significantly different momenta correspond to cosmic events (stretched shape of the cluster).

A neural network (multilayer perceptron) was created to impose a requirement on the momenta of inertia. Three momenta of inertia and the energy deposition of the biggest cluster were used as input values for the MLP. For signal training data the $n \bar{n}$ events obtained from MC simulation were used and for background training data the events from the detector with beam energy below the production threshold of the $n \bar{n}$ pair were used. An output of the MLP close to "1" means it is a $n \bar{n}$ event and close to "-1" - a background event (Fig. 3).

The following main requirements for identification of $n \bar{n}$ events were used:

- Large energy deposition in the calorimeters (900 - $1600 \mathrm{MeV}$ )

- No hits in the outer muon range system to eliminate cosmic events

- No tracks in DC coming from the beam interaction point

- Restriction for the shape of interaction in calorimeters (a requirement on the MLP output)

- Time of flight

The criterion using the MLP output ranged from 0.5 to 0.9 (Fig. 2) and it helped to suppress the number of backgrounds events by $\sim 100$ times while the signal events were suppressed just by a factor of $\sim 2$ (based on MC simulation data).

\section{Counting number of events}

Calculation of the number of $n \bar{n}$ events was performed using the time-of-flight values. To get the number of events we subtract from the number of events in the expected time window (N1) the number of events in the background time window(N3). For verification we do the same for two background windows $\left(N_{2}\right.$ and $\left.N_{3}\right)$. As a result, we got $N(n \bar{n})=N_{1}-N_{3}$ and $N($ control $)=N_{2}-N_{3}$ which should equal $\sim 0$. See Fig. 4 for more details. Doing this for several $E_{\text {beam }}$ below and above the $n \bar{n}$ threshold we expect to observe a jump after the production threshold.

\section{Verification of the algorithm}

This algorithm (especially a selection criterion that uses the MLP) needs to be verified by events which could be selected by another, well-known method. $e^{+} e^{-} \rightarrow p \bar{p}$ events could be 


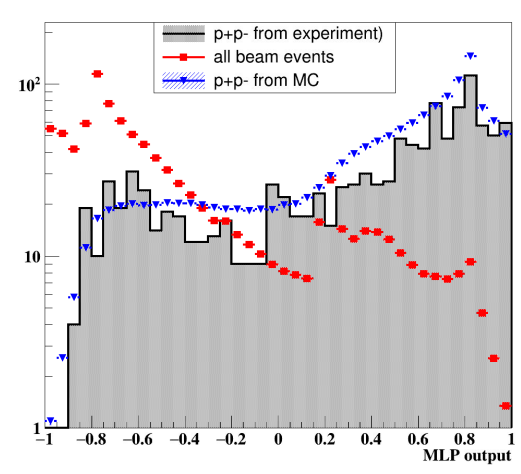

Figure 5: MLP output for $p \bar{p}$ and background events.

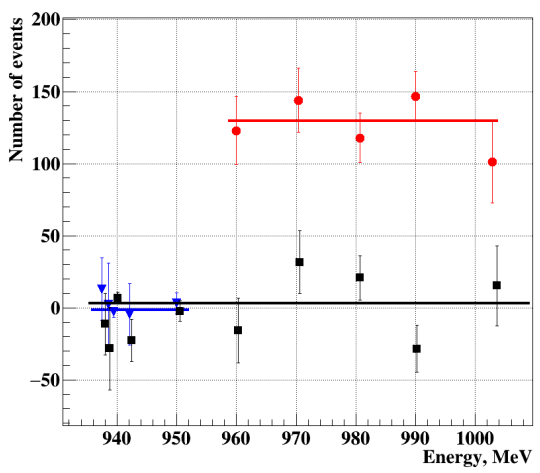

Figure 6: Counting number of $p \bar{p}$ events for different energy beam.

Blue dots $(-1.6 \pm 3.2)$ - number of $p \bar{p}$ events inside signal region by time below $E_{\text {threshold }}$; Red dots (129.5 \pm 9.2$)$ - number of $p \bar{p}$ events inside signal region by time above $E_{\text {threshold}}$; Black dots (2.9 \pm 3.1$)$ - number of $p \bar{p}$ events outside signal region by time.

easily selected by the Drift Chamber (collinear tracks with right momenta and large $\mathrm{dE} / \mathrm{dx}$ ) and could be used for verification. The energy deposition of the $\bar{p}$ differs from the value of $\bar{n}$ and new MLP needs to be trained.

Input parameters were used similar to $n \bar{n}$ : three momenta of inertia and the energy deposition of the most energetic cluster. Output parameters: "1" means $p \bar{p}$ event and "-1" means background event. For signal training data $p \bar{p}$ events obtained from MC simulation and for background training data the events from the detector with a beam energy below the production threshold of the $p \bar{p}$ pair were used (Fig. 5).

The MLP output was checked for $p \bar{p}$ events selected by the Drift Chamber and good match was observed (Fig. 5).

Counting number of events was done for several beam energies below and above the production threshold as was described above and significant "jump" of the events number was observed (Fig. 6). That leads to the conclusion that our technique works and could be applied to the identification of the $n \bar{n}$ events.

\section{Conclusion}

A technique for selecting $e^{+} e^{-} \rightarrow n \bar{n}$ events was developed and successfully tested with $e^{+} e^{-} \rightarrow p \bar{p}$ events.

We plan to improve selection criteria and try to use a deep neural network (convolutional neural network) to further suppress cosmic events. The advantage is that in this way cluster reconstruction is not used and all information from calorimeters is applied.

\section{References}

[1] A. Antonelli et al, [FENICE Collaboration], Nucl. Phys. B 517, 3-35 (1998).

[2] S.I. Serednyakov et al., Phys.Rev. D 90, 112007 (2014).

[3] B.I. Khazin et al., Nucl. Phys. B (Proc. Suppl.) 376, 181 (2008).

[4] A.N. Amirkhanov, et al., NIMPR A, in press, https://doi.org/10.1016/j.nima.2018.10.044 (2018) 